\title{
DEFLECTING FORCES FOR THE CASE OF MULTI MODE BEAM - RF CAVITY INTERACTION IN LINEAR ACCELERATORS
}

\author{
V.G.Kurakin, Lebedev Physical Institute, Leninsky Prospect 53, 117924 Moscow, Russia
}

\begin{abstract}
The formulae for the deflecting rf forces have been derived for the train of charged bunches, traversing a rf cavity. These forces originate from the deflection action of non symmetrical modes excited by the misalined bunches, the infinite set of the cavity eigen modes being taken into account. Various dependencies are explored, including the effect of "mode saturation" and non linearity of rf deflecting gradient, followed by the appropriate plots.
\end{abstract}

\section{INTRODUCTION}

High intensity proton linacs to be used in future facilities (for example, neutron spallation sources, nuclear waste transmutation etc.) assume very low beam losses to make it possible to handle such accelerators. This in turn puts forward the problems not essential for relatively low intensity machines but of vital importance for new generation of accelerators, the formation and evolution of beam halo being among them. To this end new approaches to old problems seem to be reasonable to investigate anomalies in beam dynamics. Cumulative beam instability in rf linacs [1-4] might be one of the possible mechanism contributing to beam losses. Single mode approximation traditionally used in cumulative beam break up theories can not be justified in non resonance case, if the process takes place below the regenerative threshold. Under such conditions the choice of any particular mode responsible for beam - cavities interactions becomes completely uncertain. Many of excited deflecting modes may contribute to resulting field and deflecting gradient as well, and multi mode approach suggests itself.

The paper generalises the results had been obtained in [5]. The general expression for the deflecting gradient for any bunch from the bunch train traversing the cavity is derived. Linear approximation is not used any more, because non linear character of the beam interaction with radial modes is the principle consequence of multi mode approach, determining many features of deflecting forces. Numerical calculations are performed for cylindrical resonator, followed by various plots to illustrate quantitatively multi mode beam - cavity interaction.

\section{THE EQUATIONS OF BEAM - CAVITY INTERACTION}

Our aim is the derivation of the expression for deflecting electromagnetic force produced by the charged bunches train in a cavity and experienced by the particles forming this train. It is assumed in the analysis below that there is not more than one bunch in the cavity at any moment. The influence of cavity holes on field distribution is neglected . It is assumed also that the beam is frozen in transverse direction inside cavity, so that the particle orbit is not substantially affected in its passage through the cavity. In such an approximation the electromagnetic field inside cavity acts on a particle moving with the velocity $v$ with the average transverse force [6,7]:

$$
\left\langle f_{x}\right\rangle=\frac{e v}{d} \int_{0}^{f} \frac{\partial A_{z}}{\partial x}\left(x, 0, z, \tau+\frac{z}{v}\right) d z,
$$

where $\vec{A}$ is vector potential and the integration is performed along the cavity of length $d$. (MKS units are used throughout this work.) Here, $\mathrm{x}$ is particle transverse displacement from cavity axis, $x$-axis of the basis used is assumed to coincide with it; $\mathrm{z}$ denotes the particle coordinate along the longitudinal axis, $\tau$ is the moment at which particle with charge $e$ enters the cavity.

Following [8] let us represent the vector potential in (1) in the form of infinite sum of eigenvectors $\vec{A} \lambda(\vec{r})$ :

$$
\vec{A}(\vec{r}, t)=\sum_{\lambda} q_{\lambda}(t) \overrightarrow{A_{\lambda}}(\vec{r})
$$

with the time dependent amplitudes $q_{\lambda}(t)$ satisfying the differential equation

$$
\ddot{q}_{\lambda}+\frac{\omega_{\lambda}}{Q_{\lambda}} \dot{q}_{\lambda}+\omega_{\lambda}^{2} q_{\lambda}=\frac{1}{\varepsilon_{0}} \frac{\int_{V} \vec{j} \overrightarrow{A_{\lambda}} d V}{\int_{V} \overrightarrow{A_{\lambda}{ }^{2} d V}}
$$

and with $\vec{A} \lambda$ satisfying the condition $\operatorname{div} \vec{A} \lambda=0$ as well as the Helmholtz equation:

$$
\Delta \vec{A}_{\lambda}+\frac{\omega_{\lambda}^{2}}{c^{2}} \vec{A}_{\lambda}=0 .
$$

Here, $\omega_{\lambda}$ and $Q_{\lambda}$ are frequency and quality factor of a mode respectively, $\varepsilon_{0}$ is electrical permeability of free space, $c$ is the light velocity. The solution of $(3,4)$ for the bunch of the radius $a$ and length $l$ with uniform charge $q$ distribution within it can be represented in the form:

$$
\begin{aligned}
& q_{\lambda}(t)=q \operatorname{Ir} \int_{0 D(x, a)}^{d} d S A_{\lambda, z}(x, y, z) \exp \left[\frac{\omega_{\lambda} z}{v}\left(\frac{1}{2 Q_{\lambda}}-i\right)\right] d z \times \\
& \frac{\exp \left[\omega_{\lambda}(t-\tau)\left(i-\frac{1}{2 Q_{\lambda}}\right)\right]}{\pi a^{2} \omega_{\lambda} \varepsilon_{0} \int_{V} \overrightarrow{A_{\lambda}^{2}} d V} \frac{\exp \frac{l}{v} \omega_{\lambda}\left(\frac{1}{2 Q_{\lambda}}-i\right)-1}{\frac{l}{v} \omega_{\lambda}\left(\frac{1}{2 Q_{\lambda}}-i\right)}
\end{aligned}
$$


where $D$ is the area occupied by the beam in transverse plane, and $V$ is the cavity volume. The formula is valid for the moment when the bunch has left the cavity. After passages $\mathrm{N}$ bunches, following with the time interval $T$, the induced field acts on the particles within $\mathrm{N}+1$ bunch (at the distance $\Delta l$ from the bunch head) with the force:

$$
\begin{aligned}
& \langle f\rangle=\frac{e q v}{d} \operatorname{Im} \sum_{\lambda} \sum_{n=1}^{N} Z_{\lambda}\left(x_{n}, a\right) Y_{\lambda}\left(x_{N+1}\right) F_{\lambda} \times \\
& \exp \left[\omega_{\lambda}\left((N-n+1) T+\frac{\Delta l}{v}\right)\left(i-\frac{1}{2 Q_{\lambda}}\right)\right],
\end{aligned},
$$

where the following designations are used:

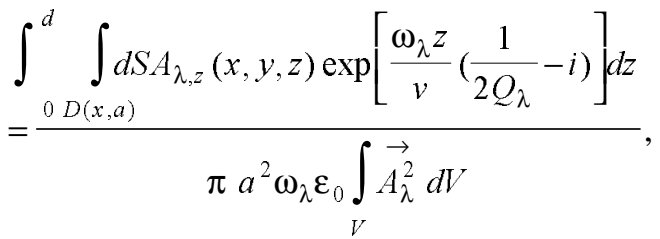

$$
\begin{aligned}
& Y_{\lambda}(x)=\int_{0}^{\partial} \frac{\partial A_{\lambda, z}}{\partial x}(x, 0, z) \exp \left[\frac{z \omega_{\lambda}}{v}\left(i-\frac{1}{2 Q_{\lambda}}\right)\right] d z, \\
& F_{\lambda}=\frac{\exp \frac{l}{v} \omega_{\lambda}\left(\frac{1}{2 Q_{\lambda}}-i\right)-1}{\frac{l}{v} \omega_{\lambda}\left(\frac{1}{2 Q_{\lambda}}-i\right)} .
\end{aligned}
$$

For steady state case formula (6) is turned to

$$
\begin{aligned}
& \langle f\rangle_{s t}=\frac{e q v}{d} \operatorname{Im} \sum_{\lambda} Z_{\lambda}(x, a) Y_{\lambda}(x) F_{\lambda} \times \\
& \frac{\exp \left[\omega_{\lambda}\left(\left(T+\frac{\Delta l}{v}\right)\left(i-\frac{1}{2 Q}\right)\right]\right.}{1-\exp \omega_{\lambda} T\left(i-\frac{1}{2 Q}\right)}
\end{aligned}
$$

\section{NUMERICAL RESULTS FOR CYLINDRICAL CAVITY}

We shall use cylindrical cavity to explore quantitatively the influence of the beam and cavity parameters on $\mathrm{rf}$ gradient for steady case state. For such a cavity the components of eigenvectors of deflecting TM modes are:

$$
\begin{aligned}
& A_{r}=-\frac{k_{z}}{k_{c}} J_{n}^{\prime}\left(r k_{c}\right) \cos n \varphi \sin k_{z} z, \\
& A_{\varphi}=\frac{k_{z} n}{k_{c}^{2}} \frac{J_{n}\left(r k_{c}\right)}{r} \sin n \varphi \sin k_{z} z, \\
& A_{z}=J_{n}\left(r k_{c}\right) \cos n \varphi \cos k_{z} z,
\end{aligned}
$$

and formula for rf gradient $\langle g\rangle_{r f}=\langle f\rangle /$ evx looks like

$$
\langle g\rangle_{r f}=\frac{I \rho}{\varepsilon_{0} d \Lambda c^{2}} G,
$$

where $I$ is the average beam current, $I=q / T$,

$G=\frac{1}{\rho^{4} \xi} \operatorname{Im} \sum_{n, m, p} \frac{v_{n, m}^{3} W_{n, m, p} S_{n, m} F_{n, m, p} \Sigma_{n, m, p} J_{n}^{\prime}\left(v_{n, m} \frac{\xi}{\rho}\right)}{k_{n, m, p} J_{n, m, p}}$

and

$$
W_{n, m, p}=-\left(i \frac{k_{n, m, p}}{\beta}-\frac{k_{n, m, p}}{2 \beta Q_{n, m, p}}\right)^{2} \times
$$$$
\frac{\left\{(-1)^{p} \exp \left(\frac{\delta k_{n, m, p}}{2 \beta Q_{n, m, p}}-i \frac{\delta k_{n, m, p}}{\beta}\right)-1\right\}}{\left(-\frac{i p \pi}{\delta}+\frac{i k_{n, m, p}}{\beta}-\frac{k_{n, m, p}}{2 \beta Q_{n, m, p}}\right)^{2}} \times
$$$$
\frac{\left\{(-1)^{p} \exp \left(-\frac{\delta k_{n, m, p}}{2 \beta Q_{n, m, p}}+i \frac{\delta k_{n, m, p}}{\beta}\right)-1\right\}}{\left(\frac{i p \pi}{\delta}+\frac{i k_{n, m, p}}{\beta}-\frac{k_{n, m, p}}{2 \beta Q_{n, m, p}}\right)^{2}} \times
$$$$
\Sigma_{n, m, p}=\frac{\exp \left[k_{n, m, p}(1+\theta / \beta)\left(i-1 / 2 Q_{n, m, p}\right)\right]}{1-\exp k_{n, m, p}\left(i-1 / 2 Q_{n, m, p}\right)}
$$

$$
F_{n, m, p}=\frac{\exp \left[\frac{\eta k_{n, m, p}}{\beta}\left(\frac{1}{2 Q_{n, m, p}}-i\right)\right]-1}{\frac{\eta k_{n, m, p}}{\beta}\left(\frac{1}{2 Q_{n, m, p}}-i\right)}
$$

$S_{n, m}=\frac{2 \rho^{2}}{v_{n, m}^{2} \pi \alpha} \int_{0}^{\psi} \cos (n \varphi) d \varphi \int_{r}^{b} J_{n}(z) z d z$, where $b=\frac{v_{n, m}}{\rho}\left(\xi \cos \varphi+\sqrt{\alpha^{2}-\xi^{2} \sin ^{2} \varphi}\right)$, $\psi=\pi, \quad r=0$ for $\alpha \geq \xi$ while $\psi=\arcsin \frac{\alpha}{\xi}$ and $\quad r=\frac{v_{n, m}}{\rho}\left(\xi \cos \varphi-\sqrt{\alpha^{2}-\xi^{2} \sin ^{2} \varphi}\right)$ for $\alpha<\xi$.

Here, lower case Greek letters designate normalised values, $R=\rho \Lambda, d=\delta \Lambda, x=\xi \Lambda, l=\eta \Lambda, \Delta l=\theta \Lambda, a=\alpha \Lambda, \Lambda$ is the wavelength of the $\mathrm{TM}_{010}$ mode, $\Lambda=c T, v_{n, m}$ is the m-th null of Bessel function of the $\mathrm{n}$-th order $J_{n}(x), \beta=v / c$, $k_{z}=\pi p / d, k_{c}=v_{n, m} / R, p=0,1, \ldots, \mathrm{m}, \mathrm{n}=1,2 \ldots$, and 


$$
\begin{aligned}
& J_{m, p}=\frac{\pi^{3} \rho^{2} p^{2}}{2 \delta v_{n, m}^{2}}\left(A_{n, m}+B_{n, m}\right)+\pi \delta C_{n, m} \times\left\{\begin{array}{l}
1, p=0 \\
\frac{1}{2}, p \neq 0
\end{array}\right\} \\
& A_{n, m}=\int_{0}^{v_{n n}} J_{n}^{2}(x) x d x, \quad B_{n, m}=\int_{0}^{v_{n n}} \frac{J_{n}^{2}}{x} d x \\
& C_{n, m}=\int_{0}^{v_{n n}} J_{n}^{2}(x) x d x, \quad k_{n, m, p}=\sqrt{\frac{v_{n, m}^{2}}{\rho^{2}}+\frac{\pi^{2} p^{2}}{\delta^{2}}}
\end{aligned}
$$

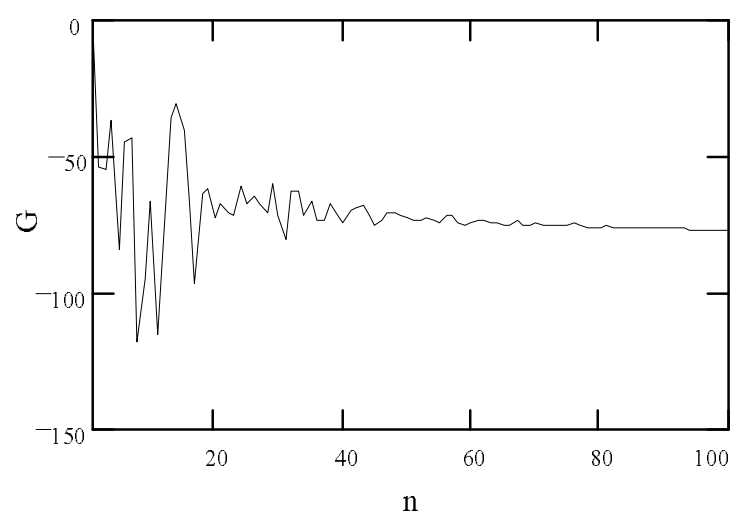

Fig. 1. Dependence of $\mathrm{rf}$ gradient on the number of modes. $\mathrm{Q}=100, \delta=0.5, \rho=0.383, \alpha=0.002, \xi=0.005, \eta=0, \theta=0$.

As numerical calculations have shown, the quadruple and higher symmetry modes contribute to deflecting gradient as small perturbations to the value, determined by dipole modes. Fig. 1 represents the dependence of resulting gradient on the modes number, $\mathrm{n}$ being equal to maximum value of radial and longitudinal modes in the sum (13). The affect of "mode saturation" is seen from the plot. The next plot (Fig. 2) illustrates the variation of rf gradient along the bunch, calculated for saturation case. Fig. 3 demonstrates strong dependence of gradient on beam offset, originating from contribution of high orders radial modes to resulting force.

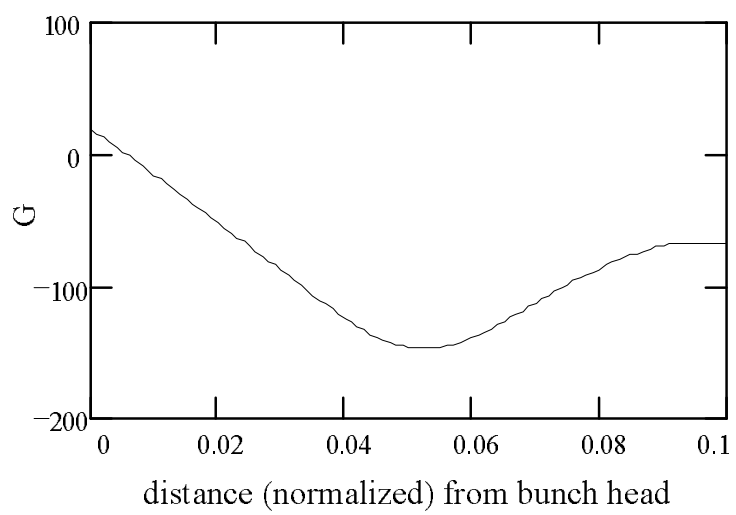

Fig. 2. Rf gradient variation along the bunch. $\mathrm{Q}=100, \delta=0.5$, $\rho=0.383, \alpha=0.002, \xi=0.005, \eta=0.05$.

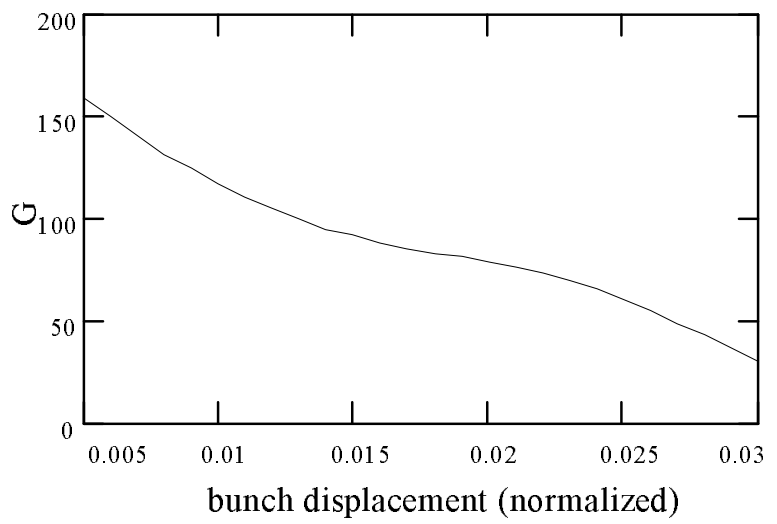

Fig. 3. Dependence of deflecting gradient on bunch displacement. $\mathrm{Q}=500, \delta=0.5, \rho=0.383, \alpha=0, \eta=0.05, \theta=0$.

\section{CONCLUSION}

Multi mode approach has been demonstrated to result in new features of beam cavity interaction in $\mathrm{rf}$ accelerators. In particular, these are strong dependence of deflecting gradient on particle location within a bunch as well as non linearity of deflecting force that manifests itself as the radial dependence of rf gradient.

\section{ACKNOWLEDGEMENTS}

The work is supported by Russian Foundation for Fundamental Research, project No 94-02-04466.

\section{REFERENCES}

[1] W.K.H.Panofsky and M.Bander, "Asymptotic Theory of Beam Break Up in Linear Accelerators", The Review of Scientific Instruments, vol. 39, pp. 206-212, February, 1968.

[2] A.W.Chao, B.Richter and C.Y.Yao, "Beam emittance growth caused by transverse deflecting fields in a linear accelerator", Nuclear Instruments and Methods, vol. 178, pp. 1-8, 1980.

[3] R.L.Gluckstern, R.K.Cooper and P.J.Channel, "Cumulative beam breakup in rf linacs", particle Accelerators, vol. 16, pp. 125-153, 1985.

[4] C.L.Bohn and J.R.Delayen, "Cumulative beam breakup in linear accelerators with periodic beam current", Physical Review A, vol. 45, No 8, pp. 5964-5993, April, 1992.

[5] V.G.Kurakin, "Multi Mode Approach in Cumulative Beam Break up Theory", in Proceedings of Fourth European Particle Accelerator Conference, London, 27 June to 1 July, 1994, pp. $1123-1125$.

[6] W.K.H.Panofsky and W.A.Wenzel, "Some Considerations Concerning the Transverse Deflection of Charged Particles in Radio-Frequency Fields", The Review of Scientific Instruments, vol. 27, No 11, p. 967, November, 1956.

[7] M.Jean Browman, "Using the Panofsky-Wenzel Theorem in the Analysis of Radio-Frequency Deflectors", in Proceedings of 1993 Particle Accelerator Conference, Washington, May 17 - 20, 1993, pp. 800 - 802.

[8] V.M.Lopuchin, The excitation of electromagnetic oscillations and waves by electron beams, Moscow: The publisher of technical and theoretical literature, 1953, 324 pp., in Russian. 